\title{
Magnetotactic Bacteria Powered Biohybrids Target E. coli Biofilms
}

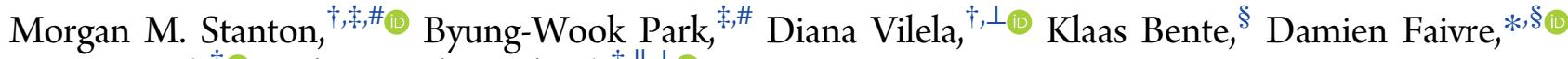

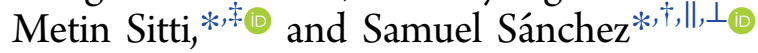

${ }^{\dagger}$ Lab-in-a-Tube and Nanorobotic Biosensors, Max Planck Institute for Intelligent Systems, Heisenbergstraße 3, 70569 Stuttgart, Germany

${ }^{\ddagger}$ Physical Intelligence Department, Max Planck Institute for Intelligent Systems, Heisenbergstraße 3, 70569 Stuttgart, Germany

${ }^{\S}$ Department of Biomaterials, Max Planck Institute of Colloids and Interfaces, Science Park Golm, Am Mühlenberg 1, 14476 Potsdam, Germany

"Institució Catalana de Recerca i EstudisAvancats (ICREA), Pg. Lluís Companys 23, 08010 Barcelona, Spain

${ }^{\perp}$ Smart Nano-Bio-Devices, Institute for Bioengineering of Catalonia (IBEC), Baldiri i Reixac 10-12, 08028 Barcelona, Spain

\section{Supporting Information}

\begin{abstract}
Biofilm colonies are typically resistant to general antibiotic treatment and require targeted methods for their removal. One of these methods includes the use of nanoparticles as carriers for antibiotic delivery, where they randomly circulate in fluid until they make contact with the infected areas. However, the required proximity of the particles to the biofilm results in only moderate efficacy. We demonstrate here that the nonpathogenic magnetotactic bacteria Magnetosopirrillum gryphiswalense (MSR-1) can be integrated with drug-loaded mesoporous silica microtubes
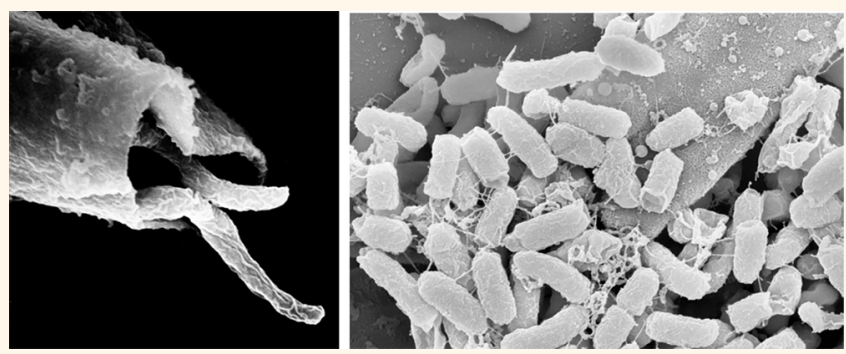
to build controllable microswimmers (biohybrids) capable of antibiotic delivery to target an infectious biofilm. Applying external magnetic guidance capability and swimming power of the MSR-1 cells, the biohybrids are directed to and forcefully pushed into matured Escherichia coli $(E$. coli) biofilms. Release of the antibiotic, ciprofloxacin, is triggered by the acidic microenvironment of the biofilm, ensuring an efficient drug delivery system. The results reveal the capabilities of a nonpathogenic bacteria species to target and dismantle harmful biofilms, indicating biohybrid systems have great potential for antibiofilm applications.
\end{abstract}

KEYWORDS: biohybrid, biofilm, magnetotatic bacteria, E. coli, antibiotic, mesoporous silica

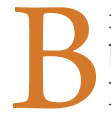

iofilms are composed of heterogeneous communities of bacteria encased in a self-extruded polymeric matrix known as exopolysaccharides (EPS), whose formation is a survival mechanism that enables bacteria to adapt and protect themselves from hostile environments, antibiotics, or the immune systems of host organisms. ${ }^{1}$ Numerous research methodologies have explored the formation of biofilms and possibilities for their eradication. Biofilms are pervasive in industrial settings, resulting in metallic corrosion or fluid blockage, ${ }^{2}$ as well as hospital environments, where they can develop on medical implants or wounds, causing devastating infections. ${ }^{3}$ An added burden is the increased resistivity of bacteria and biofilms to current antibiotics ${ }^{4}$ and their ability to develop a tolerance to antibiotics after repeated exposure. ${ }^{5}$ Consequently, the economic and social impact of biofilms is considerable, with infections being the fourth leading cause of death in hospitals in the $\mathrm{USA}^{6}$ with significant financial costs to target bacteria infections. ${ }^{7}$ One of the most common, noninvasive methods for treating clinical bacterial infections is the administration of broad spectrum antibiotics. In this fashion, the nontargeted delivery mechanism is not always successful at destroying biofilms, as biofilms are less susceptible to antibiotics when compared to planktonic bacteria. ${ }^{8,9}$ Bacteria antibiotic resistance can develop; thereupon stronger medication or surgical options are needed to remove the biofilm. Other methods for reducing the risk of localized bacterial infections include treating the surfaces of bacteria-prone areas (e.g., medical implants, tooth surfaces) with antifouling properties such as silver nanoparticles ${ }^{10}$ or antibiotic-loaded nanoparticles, ${ }^{11}$ but these surface treatments can only target biofilm at the material interface and over time can become inert due to protein adhesion. ${ }^{12}$ Unbound liposomes ${ }^{13}$ and nanoparticles ${ }^{14}$ have also shown promise for drug delivery to treat

Received: June 13, 2017

Accepted: September 21, 2017

Published: September 21, 2017 

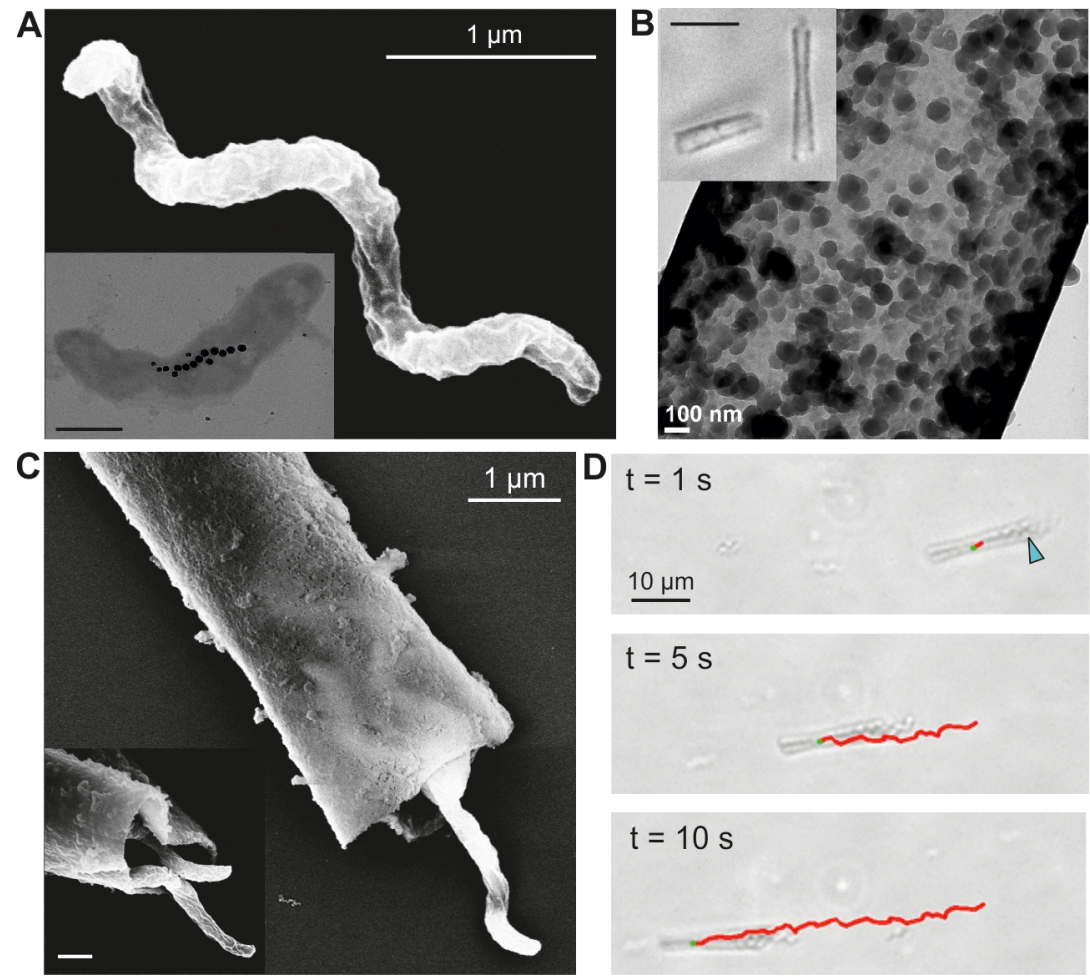

Figure 1. Characterizing bacteria-driven biohybrid microswimmers. (A) SEM image of MSR-1 bacterium. Inset displays TEM of MSR-1 and internal magnetosome chain. Inset Scale bar $=500 \mathrm{~nm}$. (B) TEM image of pores in MSMs. Inset displays bright field microscopy images of long and short MSMs. Inset scale bar $=10 \mu \mathrm{m}$. (C) SEM of MSR-1 cells captured within a microtube. Inset displays increased magnification of bacteria in tube. Inset scale bar $=500 \mathrm{~nm}$. (D) Bright field microscopy images of MSR-1-powered biohybrid swimming. Blue arrow indicates location of MSR-1 inside the microtube. Red track indicates the trajectory of the biohybrid.

biofilms or cancer; however these targeted measures are limited by their dependence on host circulation and serendipitous encounters with biofilm interfaces, limiting their potency.

Micro- and nanoswimmers offer an alternative and capable method for targeted drug delivery, ${ }^{15,16}$ and the fluidic propulsion that bacteria create can be an optimal energy source for biohybrid swimmers. ${ }^{17,18}$ Biohybrid swimmers merge active motile cells with an artificial material, where the cells simultaneously act as bioengines and biosensor $(\mathrm{pH}$, temperature, chemical) and the artificial cargo load can serve as the drug delivery device. ${ }^{19}$ The artificial and cellular components of the biohybrids form an active swimming system with greater navigation and sensing capabilities than monocomponent swimmers (either biological or artificial) or magnetic particles. $^{20-22}$ Recent biohybrid microsystem publications have utilized sperm cells, ${ }^{23,24}$ contractive muscle cells, ${ }^{25-27}$ and bacteria ${ }^{28,29}$ to create micro- or macroscale actuators to perform work for potential biomedical applications, but implementing biohybrids for biofilm treatments has not yet been explored. Here, we present magnetotactic bacteria biohybrid microswimmers for antibiotic delivery to biofilms that address problems with current antibiofilm agents. Earlier biohybrid work using magnetotactic bacteria propulsion has proven bacteria are capable of micromanipulation, ${ }^{30}$ microselfassembly, ${ }^{31,32}$ and carrying cargo loads in microfluidic devices. ${ }^{33}$ More recently, the magnetotactic bacteria Magnetococcus marinus (MC-1), were used to power biohybrids for in vivo tumor targeting in mice, indicating magnetotactic bacteria have potential for clinical applications after further immune response studies. ${ }^{34}$ For the locomotive component or the presented biohybrid, the magnetotactic bacteria Magneto- sopirrillum gryphiswalense, MSR-1, were chosen to power the swimmer due to their capacity to sense and respond to their surrounding environment, ability to be externally guided with low magnetic fields, and rapid and high-density culture cycle. ${ }^{35}$ MSR-1 bacteria swim using rotating bipolar flagella and contain a chain of self-produced magnetosome nanoparticles composed of $\mathrm{Fe}_{3} \mathrm{O}_{4}$ (Figure 1A) that assist in directing the cells to preferred oxygen concentrations in their native aquatic enviornments. ${ }^{36}$ MSR-1 cells were strategically adhered inside mesoporous silica microtube (MSM) structures that had been preloaded with the antibiotic ciprofloxacin (CFX). Mesoporous silica is biocompatible and has a high surface area that is apt for drug storage and delivery, ${ }^{37}$ and the tubular structure of the chassis has been shown in our previous report to be a beneficial configuration for improved bacteria biohybrid directional swimming. ${ }^{38}$ The MRS-1-CFX-MSM biohybrid system was capable of being magnetically guided to E. coli biofilms, penetrating into the biofilm, and releasing the CFX cargo load, triggered by the low $\mathrm{pH}$ of the biofilm microenvironment.

\section{RESULTS}

Formation and Characterization of Biohybrids. The tubular, mesoporous silica chassis of the biohybrid was fabricated using an organic synthesis method with a commercially available polycarbonate (PC) membrane template. Mesoporous silica formed within the $2 \mu \mathrm{m}$ pores of the template, and after polishing, mesoporous silica microtubes were released by chemical solvation of the template and subsequent washing and sonication (Figure 1B). The conical pores of the membrane formed the asymmetric shape of the MSMs, and the MSMs were produced in two lengths, long 

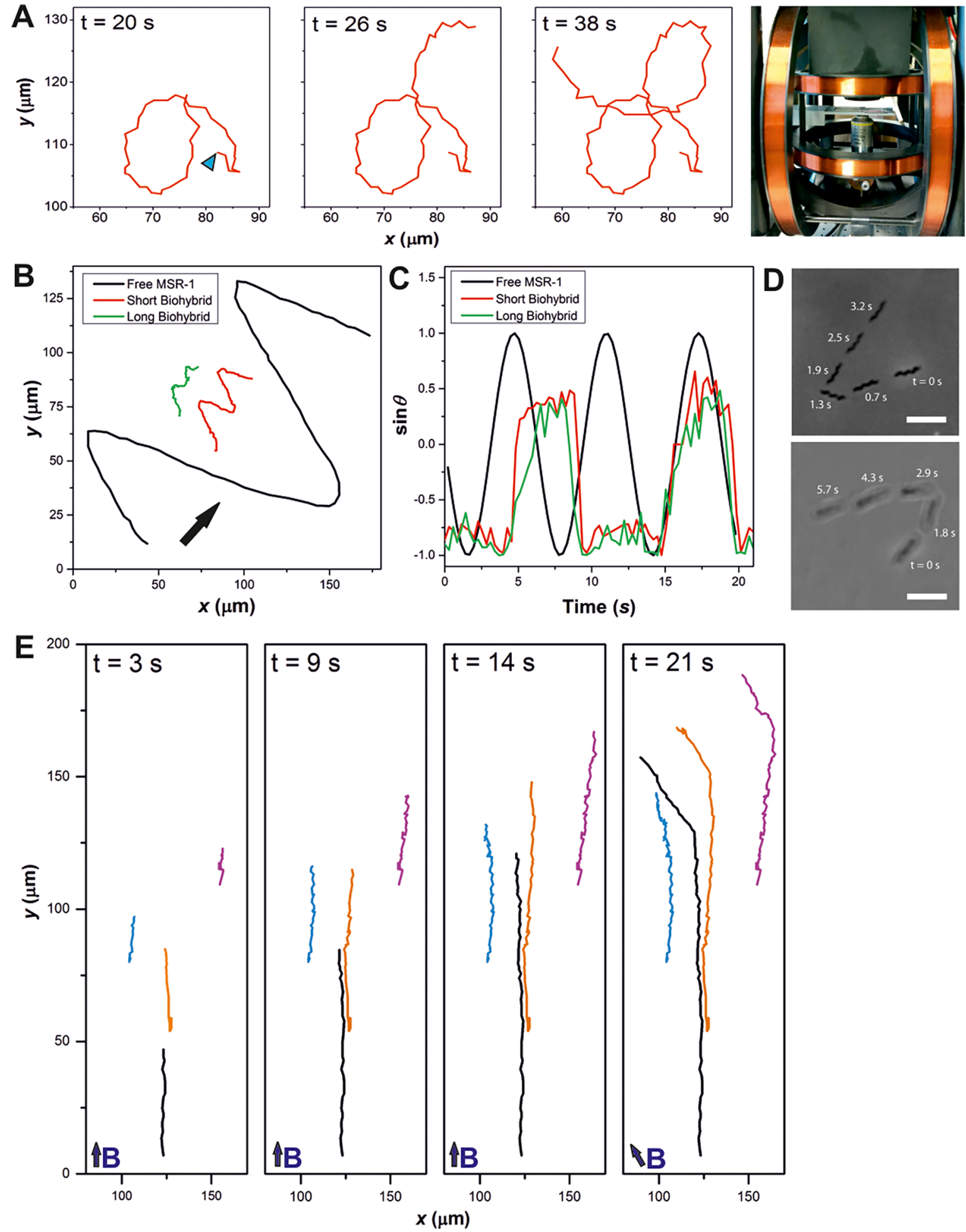

Figure 2. Magnetically guided swimming. (A) Track of a single biohybrid (left) guided with an external magnetic coil system (right). The starting point of the track is indicated with a blue arrow. (B) Trajectories of free MSR-1 cells or biohybrids moving in a periodically reversing magnetic field. Arrow indicates the general direction of swimming. (C) Sine of the angle $(\sin \theta)$ of the cell or biohybrid in the rotating magnetic field. (D) Time lapsed U-turn analysis of MSR-1 (top) and biohybrid (bottom). (E) Tracks of four separate biohybrids with magnetic guidance.

( $\sim 20 \mu \mathrm{m}$ in length) and short $(\sim 10 \mu \mathrm{m}$ in length), where short MSMs were due to breaking of single long MSMs. Transmission electron microscopy (TEM) images of the MSMs show nanoscale pores distributed throughout the tube body. After characterization of the MSMs, biohybrids were created by incubating MSR-1 with MSMs in a buffer solution for 15 min with gentle shaking to promote MSR-1 encounters and adhesion with the tubes. MSR-1 adhered inside the tube and were capable of swimming with the tube over prolonged durations. Scanning electron microscopy (SEM) images in 

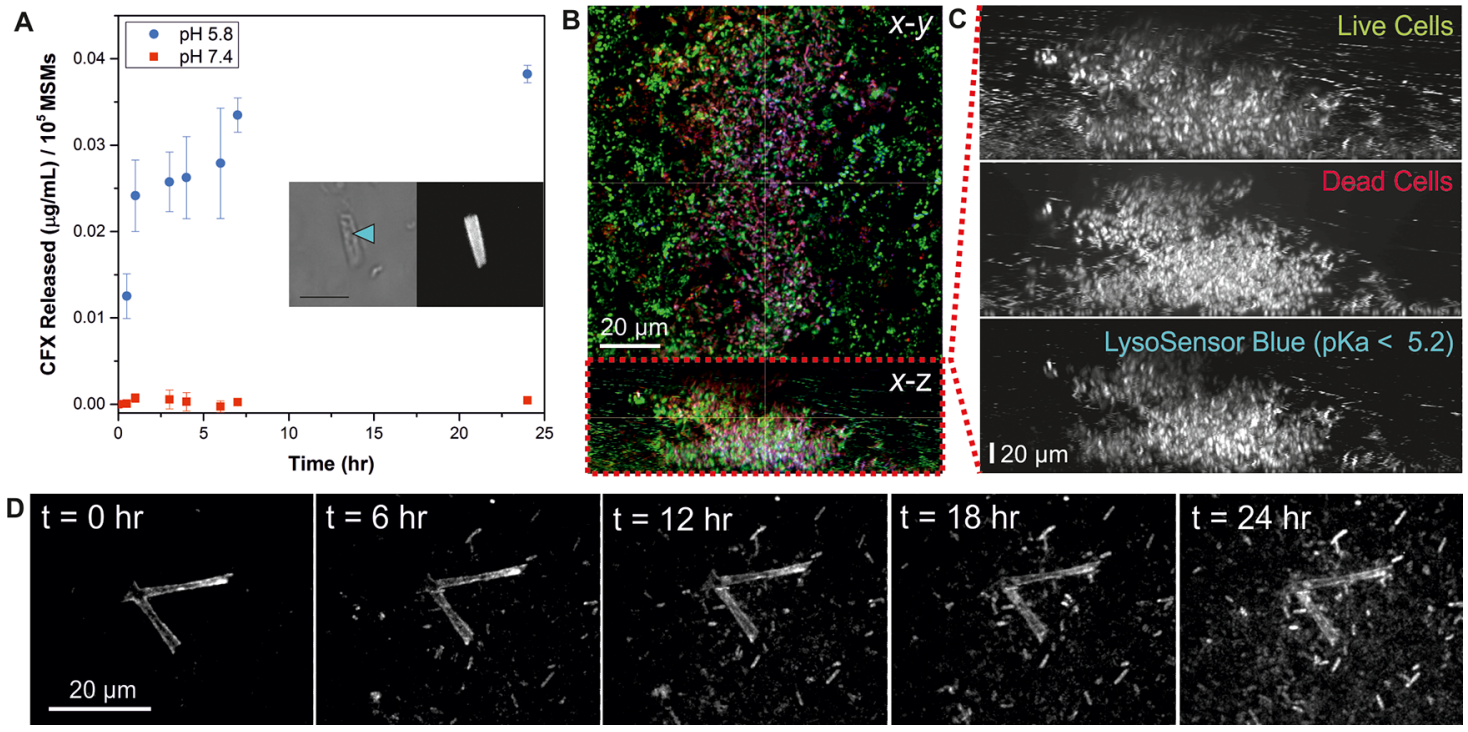

Figure 3. $\mathrm{pH}$-triggered release of CFX antibiotics from CFX-MSMs. (A) CFX release over time from CFX-MSMs at pH 5.8 and 7.4. Error bars indicate the SD of the mean $(n=6)$. Inset displays MSR-1 swimming with a CFX-MSM. (Left) Bright field image of MSR-1 (blue arrow) swimming with an MSM and (Right) fluorescence image of CFX loaded in the tube. Inset scale bar $=10 \mu \mathrm{m}$. (B) Confocal imaging of live cells (green), dead cells (red), and localized acidic environments (blue) within an $E$. coli biofilm. (C) The $x-z$ plane from (b) is separated into individual fluorescence panels. Acidic microenvironments were labeled with the $\mathrm{pH}$-sensitive dye, LysoSensor Blue (fluoresces only when $\mathrm{pK}$ < 5.2). (D) Compressed confocal stacks of CFX-MSMs biohybrids in an E. coli biofilm over $24 \mathrm{~h}$. Observed fluorescence is due only to CFX.

Figure 1C display MSR-1 partially penetrating MSMs. The flagella of the MSR-1 with or without the tube could not be observed, but it is hypothesized that the flagella motion of MSR-1 is similar to Spirillum volutans bacteria, where the rotating flagella cause the cell body to rotate in the opposite direction, eliminating torque. ${ }^{39}$ Once the MSR-1 are within the MSM, it is unknown if both flagella or only a single flagella are rotating, but the biohybrids exhibit continuous directional motion. Specific bacteria adhesion inside the tubes was controlled by providing amine chemical moieties only inside the MSM structure (Supporting Information, Figure S1). Limited studies have examined the adhesion of magnetotactic bacteria to surfaces, but the positive charge of the surfacebound (3-aminopropyl)triethoxysilane (APTES) may promote bacteria adhesion due to van der Waals and electrostatic forces. ${ }^{12}$ APTES localized within the MSMs created guided cell adhesion generating unidirectional forces from the bacteria flagella and allowing the biohybrid to swim efficiently (Figure 1D). The choice of using a magnetically guidable bacteria instead of a magnetically guidable microtube chassis was based upon the multiple capabilities of the MSR-1: swimming, sensing, and magnetic sensitivity. This permitted a larger variety of materials for the tube chassis, materials such as mesoporous silica, which is ideal for drug storage and delivery. As mentioned in our previous article, addition of magnetic components to the chassis increased the density of the microtube; bacteria were still capable of attaching to the microtube, but most biohybrids were not able to swim with the heavier load. ${ }^{38}$ Use of MSR-1 bacteria resolves this issue.

Once the biohybrid was formed, the microswimmer could be magnetically guided using a permanent magnet (Video SV1) or using electromagnetic coils as seen in Figure 2. The magnetic field, $B$, induced a torque that passively aligned the MSR-1 along the magnetic field lines, forcing the bacteria to swim in a single direction. Biohybrids were capable of following constant changes in the magnetic field and could be directed to swim in complex microtrajectories, such as a figure-eight (Figure 2A,
Video SV2), demonstrating they can operate in microenvironments and be guided to biofilm locations. To further investigate their swimming trajectories under magnetic fields, free swimming MSR-1, short biohybrids, and long biohybrids were observed swimming in a periodically reversing magnetic field using the "U-turn method" ${ }^{40}$ By reversing a $6 \mathrm{mT}$ magnetic field every $6 \mathrm{~s}$, MSR-1 cells responded by changing their swimming direction and completing a U-turn. The U-turn method is often used to find the magnetic moment of magnetotactic bacteria, ${ }^{41}$ but it can also be used to analyze the performance of a magnetically guided biohybrid. Figure $2 \mathrm{~B}$ displays examples of trajectories of the cells and biohybrids in the switching field. The velocities of the biohybrids, long $(4 \pm 1$ $\mu \mathrm{m} / \mathrm{s})$ and short $(8 \pm 1 \mu \mathrm{m} / \mathrm{s})$, are slower than the free bacteria and cause the length and diameter of their U-turn trajectories to be much shorter, with the longer biohybrids having U-turns with the lowest diameters. The free-swimming MSR-1 have a higher velocity $(21 \pm 5 \mu \mathrm{m} / \mathrm{s})$, so they are capable of swimming larger distances between each field reversal and have a greater diameter in their U-turn trajectory compared to the biohybrids. The sine of the angle $(\sin \theta)$ of the free bacteria or biohybrid relative to the $x-y$ plane was also measured over time (Figure $2 \mathrm{C}$ ) and assisted in visualizing the duration of the U-turns. The free bacteria responded almost instantaneously to the reversal in magnetic field; however the biohybrids needed greater time in reversing their direction (Figure 2D). The delay in changing direction is attributed to the increased force required for the MSR-1 to rotate the tube, but they are still capable of rotating the MSM $180^{\circ}$ within the 6 $\mathrm{s}$ window. Figure $2 \mathrm{~B}$ and $\mathrm{C}$ provide useful insight on biohybrid rotation capabilities and response time within the magnetic field. The diminished U-turn diameters and the slower turning times of the long biohybrids indicate that there is an upper limit to the length of the MSM that is capable of being reoriented by MSR-1 in a magnetic field. Although longer MSMs would be capable of carrying larger drug doses, there would be a small sacrifice in maneuverability. Shorter biohybrids would be more 

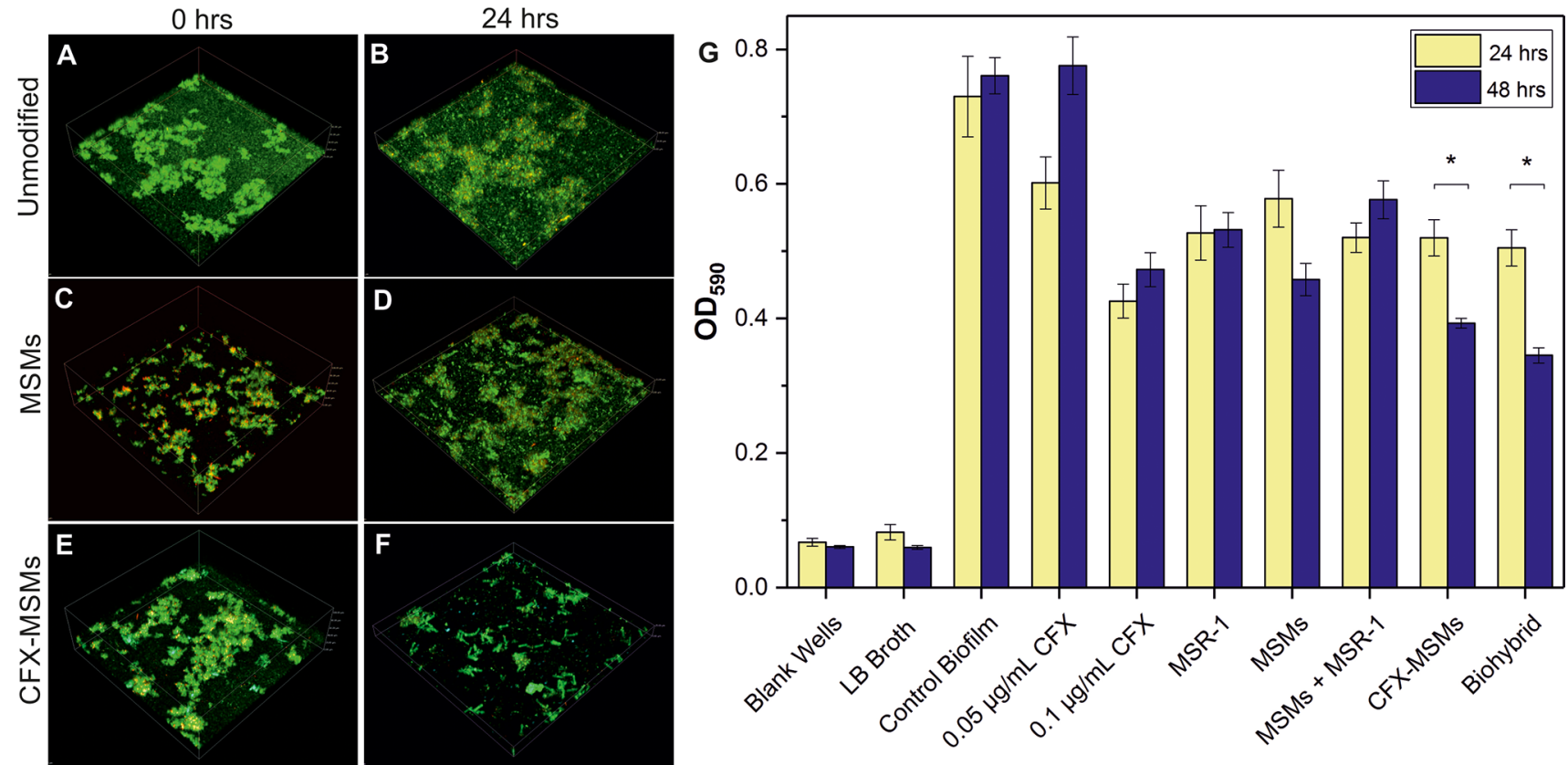

Figure 4. E. coli biofilm disruption. Overnight cultured biofilms had live (green) and dead cells (red) compared in $350 \times 350 \mu \mathrm{m}^{2} 3 \mathrm{D}$ confocal images. (A, B) Unmodified control biofilms, (C, D) biofilms with MSMs, and (E, F) biofilms with CFX-MSMs were compared at the time of incubation with the microtubes $(0 \mathrm{~h})$ and $24 \mathrm{~h}$ later. CFX fluorescence is shown in e and $\mathrm{f}$ in cyan. (G) CV absorbance analysis of biofilms after 24 or $48 \mathrm{~h}$ of incubation with biohybrids (CFX-MSMs+MSR-1) and control groups. Error bars indicate the SD of the mean $(n=15)$, and statistical significance (with $P<0.01$ ) is indicated with * for data sets that are significantly different between the 24 and 48 h time points.

advantageous for guidance to microscale or mobile targets. This information will be essential for future development of bacteriapowered biohybrids and in vivo applications.

Coordinating biohybrid swimming is recognized as a significant challenge in the field of biohybrids, as a significant number of the biohybrids are needed to deliver therapeutic doses of medicine. We acknowledge similar difficulties with the presented microswimmer platform, as not every MSM captures a bacterium. Currently we cannot separate biohybrids from free swimming bacteria, making it difficult to quantitatively confirm the yield of success to biohybrid form. However, there has been success in collecting and monitoring the swimming behavior of small groups of biohybrids. Using an external magnetic field, clusters of biohybrids were observed swimming and operating together. An example is shown in Figure 2E (Video SV3), where four biohybrids swim in unison in the direction of the magnetic field. Unified group swimming of large quantities of biohybrids is recommendable for macroscale targets, such as tumors, ${ }^{34}$ but small clusters of biohybrids have an advantage for aiming at smaller targets, such as biofilms. Infectious biofilms are mobile and heterogeneous with scale sizes in the micrometer range. Instead of overwhelming the drug delivery target with biohybrids to increase efficacy, biohybrids may also operate in a "stealth" mode, where antibiotic can be delivered to precise locations to do the most damage. Targeted delivery of antibiotics and biofilm disruption is discussed further in later results.

CFX and CFX-MSMs with Bacteria and Biofilms. The fluoroquinolone antibiotic CFX was chosen to load the MSMs for four major reasons: (1) CFX is a clinically relevant antibiotic and is prescribed for numerous bacterial infections, (2) the low dose of antibiotic needed for the minimum inhibitory concentration (MIC) for E. coli, ${ }^{42}$ (3) the solubility of CFX is dependent on solution $\mathrm{pH}$, and (4) the fluorescence of the antibiotic is in the visible spectrum, providing a facile method for detection and concentration analysis. MSR-1 and $E$. coli behavior and viability in the presence of CFX are discussed in the Supporting Information (Figure S2). To load MSMs with CFX, MSMs were incubated in an aqueous acidic CFX solution overnight and then washed to produce CFX-MSMs. The solubility of CFX in aqueous solutions is $\mathrm{pH}$ dependent, only becoming soluble in acidic solutions, so the release of CFX from CFX-MSMs was meaured at two different $\mathrm{pH}$ 's in motility media, 5.8 and 7.4 (Figure 3A). At pH 7.4, almost no CFX was released from the tubes over $24 \mathrm{~h}$, but there was a significant increase in release when the $\mathrm{pH}$ of the soltuion was lowered to 5.8. After $24 \mathrm{~h}, \sim 0.04 \mu \mathrm{g} \mathrm{mL} L^{-1}$ of CFX had been released from $10^{5}$ MSMs, illustrating that $\mathrm{pH}$ could be used as a trigger to release the antibiotic from the MSMs. As biofilms have previously shown localized acidic microenviornments within the biofilm, ${ }^{43,44}$ they are an ideal spark to initiate CFX release from CFX-MSMs. In Figure 3B, an E. coli biofilm had live cells (green), dead cells (red), and acidic microenviornments of cells and EPS (blue) fluorescently labeled. Acidic regions were labeled with a $\mathrm{pH}$-sensitive probe, LysoSensor Blue DND-167, which is essentially nonfluorescent when the $\mathrm{p} K_{\mathrm{a}}>5.2$. It is observed in the black and white $x-z$ cross sections of the biofilm image (Figure 3C) that the LysoSensor dye stained a significant portion of the interior of the biofilm, indicating a localized acidic microenivornment which included live and dead MSR-1. To ensure these observed acidic pockets in the biofilm would trigger CFX relase from CFX-MSMs, CFX-MSMs were incubated in $48 \mathrm{~h} \mathrm{E}$. coli biofilms and had their CFX fluorescence monitored over time with confocal microscopy (Figure 3D, Video SV4). After $6 \mathrm{~h}$ it is noted that surrounding bacteria in the biofilm also become fluorescent as they absorb the released CFX from the CFX-MSMs. At $24 \mathrm{~h}$, a significant portion of the surrounding biofilm and bacteria are fluorescent, revealing the biofilms are suitable for triggering release of the antibiotic from CFX-MSMs. 

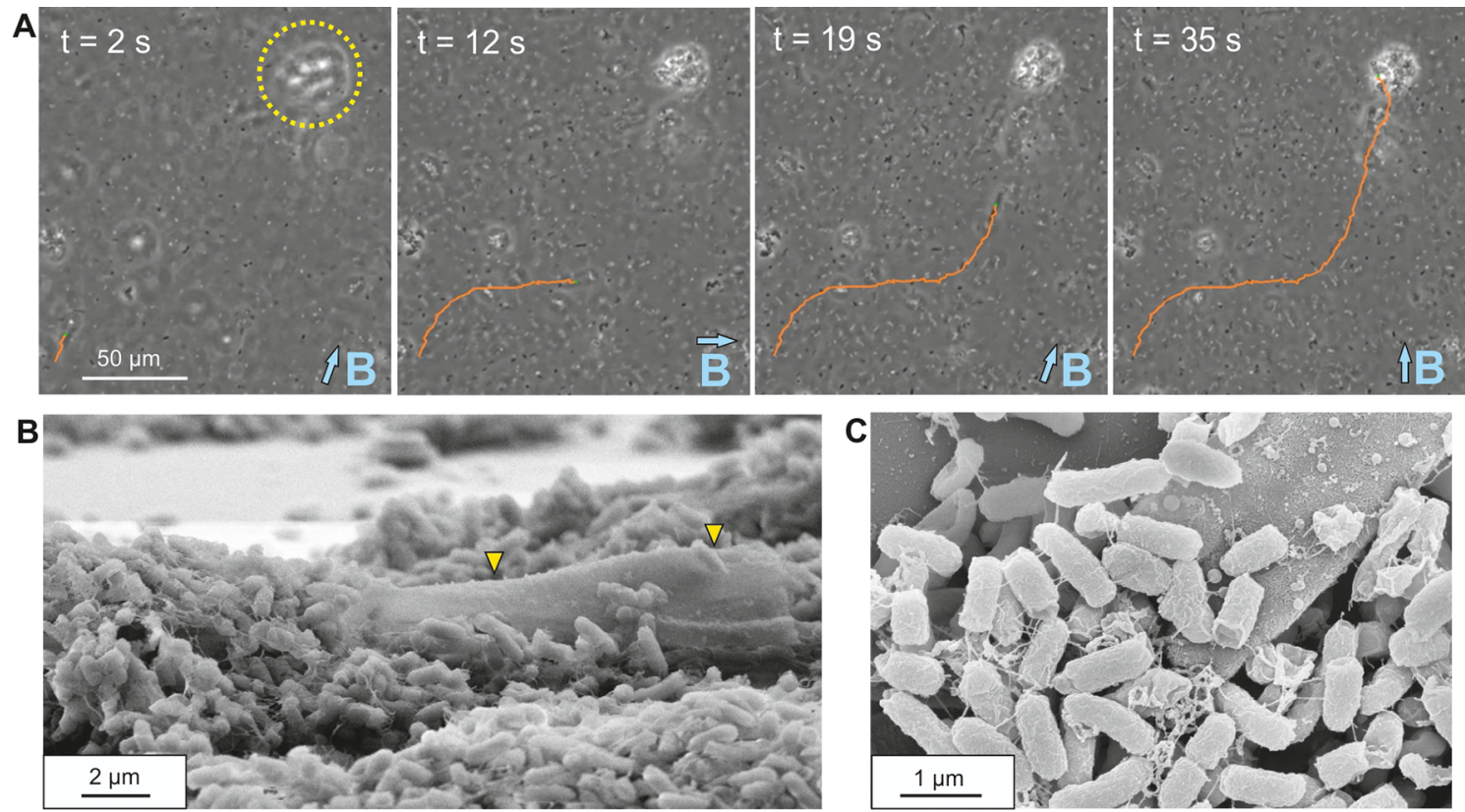

Figure 5. Magnetic guidance of biohybrids to E. coli biofilms. (A) Single MSR-1-powered biohybrid magnetically guided to an island of $E$. coli biofilm (yellow circle). The biohybrid is pushed into the biofilm and becomes trapped. (B) SEM image of a biohybrid trapped in an E. coli biofilm. Yellow arrows indicate the location of the MSM. (C) Increased magnification displays EPS and bacteria surrounding the biohybrid.

Biofilm Disruption with Biohybrids. E. coli biofilms were cultured for $24 \mathrm{~h}$ on glass coverslips, washed with motility media, and then left unmodified, incubated with MSMs, or incubated with antibiotic-loaded CFX-MSMs for another $24 \mathrm{~h}$. Live/dead cell staining was performed at the moment of microtube incubation $(0 \mathrm{~h})$ or $24 \mathrm{~h}$ after (Figure $4 \mathrm{~A}-\mathrm{F}) .3 \mathrm{D}$ confocal imaging of the biofilms displays clusters of $E$. coli on the surface. Unmodified biofilms displayed no visual change over a $24 \mathrm{~h}$ period (Figure 4A,B), and biofilms incubated with MSMs also had limited variations in bacteria density (Figure 4C,D), but biofilms incubated with CFX-MSMs had a significant change in biofilm structure after $24 \mathrm{~h}$ (Figure 4E,D). CFX-MSMs biofilms exhibited a decrease in clustering after incubation with the antibiotic tubes, and final bacteria density was visibly lower compared to the $0 \mathrm{~h}$ time point. To further quantify the effect of the biohybrids on the $E$. coli biofilms, crystal violet (CV) staining was employed. The 96well plates of $24 \mathrm{~h}$ cultured biofilm were washed and incubated with the proposed biohybrid (MSR-1+CFX-MSMs) or nine other control groups for 24 or $48 \mathrm{~h}$ as seen in Figure 4G. After incubation with the biohybrid and control groups, biofilms were stained with $\mathrm{CV}$, and the optical density (OD) at $590 \mathrm{~nm}$ $\left(\mathrm{OD}_{590}\right)$ was measured to quantify the concentration of $E$. coli biofilm. Exposing the biofilms to $0.05 \mu \mathrm{g} \mathrm{mL}^{-1}$ CFX solutions resulted in insignificant disruption of the biofilm at both the 24 and $48 \mathrm{~h}$ incubation time points, while a higher concentration of CFX, $0.1 \mu \mathrm{g} \mathrm{mL}^{-1}$, provided a $\sim 40 \%$ decrease in CV biofilm absorbance after $24 \mathrm{~h}$ that remained constant at $48 \mathrm{~h}$. The increased concentration is significantly higher than the CFX concentration needed to reduce planktonic E. coli proliferation, but the result is expected, as biofilms have been shown to be less susceptible to antibiotics than free-swimming bacteria. Biofilms incubated for $24 \mathrm{~h}$ with MSR-1, MSMs+MSR-1, CFXMSMs, and biohybrids also displayed decreases in biofilm formation when compared to the unmodified control biofilm, with biohybrids inducing a $32 \%$ decrease in CV absorbance. It is interesting to note that the MSR-1 bacteria and MSMs
+MSR-1 also produced similar reductions in absorbance without the assistance of antibiotic after $24 \mathrm{~h}$. We hypothesize that the locomotion and consumption of resources (nutrients, oxygen) of large quantities of MSR-1 bacteria have the capability to disturb the biofilm and prevent static biofilm development as well, therefore decreasing the CV absorbance. However, there is no significant change between the 24 and 48 $\mathrm{h}$ time points for control groups containing MSR-1, indicating they would only be useful for temporarily disrupting foreign biofilm. MSR-1 motility decreases over time and ceases once the bacteria die and their death is accelerated at physiological temperatures, leaving MSR-1 inactive 30 to $45 \mathrm{~min}$ after exposure to $37{ }^{\circ} \mathrm{C} .{ }^{45}$ Only the CFX-MSMs group and biohybrid group (MSR-1+CFX-MSMs) demonstrated a statistically significant decrease in CV absorbance between the 24 to $48 \mathrm{~h}$ time point (25\% and $32 \%$ decrease, respectively, labeled with an asterisk in Figure 4G). The biohybrid group also had the greatest overall decrease in $\mathrm{CV}$ absorbance from the unmodified biofilm control group at $48 \mathrm{~h}$ (55\%) when compared to the other controls. In the presence of only antibiotic solution or MSR-1 bacteria, the E. coli biofilm disruption plateaus after $24 \mathrm{~h}$, but the antibiotic-loaded biohybrid swimmer has an increased antibiofilm effect that continues to disrupt biofilm until $48 \mathrm{~h}$, displaying biohybrids' therapeutic advantages as an active drug delivery system.

To prove the overall advantage of our biohybrid systems over current biofilm disruption methodologies, we demonstrated our swimming biohybrids targeting and delivering their antibiotic cargo load. Biohybrids powered by MSR-1 were incubated with E. coli biofilms. Using a permanent magnet, biohybrids were guided through concentrated E. coli populations to clusters or islands of dense biofilm (Figure 5). Multiple biofilm regions were targeted in a single Petri dish using different biohybrids (Figures 5A and S3), showing the benefits of operating low numbers of biohybrids to attack multiple microscale targets. Once the target biofilm clusters were reached, the biohybrids could be pushed into the biofilm using the force of the MSR-1 
propulsion (Video SV5). Although the power of the MSR-1 provides energy to push into the biofilm, once inside, it becomes trapped within the biofilm matrix, becoming incapable of further motility (Figure 5B,C). The biofilm EPS is the largest contributor to the viscoelastic properties of biofilm and has been shown to deform or rearrange its structure due to external stress, such as applied pressure or shear forces. ${ }^{1}$ Penetration of the biohybrid into the biofilm causes a temporary elastic deformation, but the high viscosity of the biofilm prevents further biohybrid swimming. This delivery and capture mechanism is an efficient method to ensure targeted drug delivery to the acidic interior biofilm environments. Combining localized cell adhesion for propulsive force, MSR-1 magnetic sensing, and MSM drug loading capacity, we have presented a biohybrid with the potential to disrupt mature E. coli biofilms.

\section{DISCUSSION}

Even after decades of research, an in-depth understanding of biofilm formation and eradication methodologies remains elusive. There are still many questions regarding the biofilm EPS composition, bacteria communication within the biofilm (quorum sensing), and biofilm metabolic pathways to resist antibiotics. ${ }^{1,7}$ To engage with such a complex biological system, a radical alternative to current methods needs to be presented. The MSR-1-propelled biohybrid demonstrates the feasibility of using a "hero" nonpathogenic bacteria to deliver antibiotics to infectious biofilms. The MSR-1 bacteria possesses the ability to sense and respond to external magnetic fields, allowing the biohybrid to have targeted antibiotic delivery to motile biofilm cites and the ability to penetrate the microtubular systems deep within the biofilm body and EPS, where the drug release is triggered by biochemical properties of the biofilm. Although this article has demonstrated a proof-of-concept of application, there are still many steps forward until such bacteria biohybrids may be used in vivo. ${ }^{46}$ Other types of synthetic swimmers, such as helical nanopropellers ${ }^{20}$ or micro artificial bacterial flagella, ${ }^{21}$ do not require bioenergy propulsion, but swim by rotation using an external rotating magnetic field. These swimmers could also be considered for the task of antibiotic delivery, as they offer accurate guidance systems with proven drug delivery capabilities. However, these systems lack the sophisticated sensing mechanisms of biological organisms. Helical microswimmer systems require visual observation to be guided toward their intended target, diminishing their applicability for drug delivery to concealed locations until there are improved imaging and resolution techniques. Free swimming bacteria have also shown to disrupt biofilms by tunneling through the matrix, allowing toxic chemicals to permeate the biofilm, ${ }^{47}$ but there is no navigational control of the tunneling bacteria, making it difficult to target a precise location. To achieve future in vivo targeted drug delivery, microswimmers will require multicomponent guidance systems utilizing external magnetic control to reach a general location as well as more sensitive local sensing, such as $\mathrm{pH}$, temperature, or biochemical guidance, to find specific microenvironments. Bacteria biohyrids incorporate a synthetic component for triggered drug delivery and a bacterium as a living power source and natural sensor, giving them an advantage over single-element synthetic or cellular swimmers. The presented MSR-1-powered biohybrid moves the microswimmer field closer toward clinical applications and illustrates that bacteria biohybrids can be employed for antibiofilm research.
In addition to our biohybrid design properties to target biofilms, we have also considered the effect of our biohybrid on nonpathogenic host bacteria. The plethora of varied bacteria in the human microbiome has only recently been investigated, and initial research has shown bacteria colonies around the human body form symbiotic relationships with their hosts. These relationships have proven essential to maintain good health, as the natural microflora prevent the overgrowth of opportunistic and pathogenic microorganisms. ${ }^{48}$ Antibiotics, including CFX, have shown to eliminate natural intestinal microbiota, which results in health problems including alterations in metabolism. ${ }^{49}$ Although the MIC of CFX required for E. coli has shown to be $<0.06 \mu \mathrm{g} / \mathrm{mL}$, patients with bacterial infections are given oral CFX doses of 250 to $750 \mathrm{mg}$ tablets, leading to some adverse gastrointestinal and nervous system reactions. ${ }^{42,50}$ Guided manual delivery of antibiotics could also improve the medical side-effects of nontargeted antibiotics and help maintain natural host bacteria. As shown in Figure 4G, the antibiotic-loaded biohybrids have a greater effect at reducing established biofilm when compared to CFX solutions. By guiding the biohybrids to defined locations, the potency of the antibiotic will only be realized at the infection site. Antibiofilm agents that include protection of natural benign bacteria improve the efficacy of drug delivery, by localizing bacteria damage and reducing indirect health complications.

Therapeutic methods could also benefit from the penetration delivery method of the presented biohybrid. Biofilm survival is directly linked with its mechanical strength and low metabolic activity. The biofilm and EPS create a protective shell forming a physical barrier for the biofilm, and the diminished growth rate of biofilms limits uptake of local antibiotics. Previous research with fluoroquinolones has shown the drugs are capable of penetrating entire biofilms, but even with prolonged exposure to antibiotics such as CFX, cell death in biofilms is observed only at the biofilm interface, while the bulk remains viable and intact. $^{51}$ The ability for $E$. coli biofilms to remain viable in antibiotics is attributed to their efflux pump systems. Efflux pump genes are highly upregulated during biofilm growth, and efflux pumps are responsible for waste and toxic substance removal, including antibiotics, resulting in antibiotic-tolerant biofilms. $^{52}$ However, physical disruption and breakage of biofilms makes biofilm bacteria more susceptible to antibiotics and therefore easier to treat. ${ }^{9,22}$ Using magnetic actuation, magnetotactic bacteria have demonstrated applied mechanical force on biofilms, resulting in subsequent biofilm damage. ${ }^{53}$ Combining physical disruption with antibiotic release would improve the therapeutic effect of antibiofilm agents. The penetration power of the MSR-1-powered biohybrid pushes through the outer shell of the biofilm and accesses the lowmetabolic cells within the biofilm core (Figure 5). This drug delivery method is effective because it utilizes a potent fluoroquinolone antibiotic and physically damages the biofilm. It could be envisioned that future therapeutic applications could use a combination of administered free antibiotic to kill planktonic bacteria and biohybrids to strike and disrupt biofilms. The two-pronged approach would be effective at eradicating robust biofilm cultures that are not affected by antibiotics alone.

\section{CONCLUSIONS}

Biohybrids operate as intelligent micro robotic systems, with a powerful bioengine and triggered antibiotic delivery. The presented biohybrids are active drug delivery systems using a 
living bacteria power source with a natural magnetic sensor and demonstrate the feasibility of an antibiofilm biohybrid. This report demonstrates the advantages of microswimmer technology for the clinically relevant and considerable problem of biofilm formation and its potential for having greater targeting and therapeutic capabilities than monocomponent swimmers or passive drug delivery. Future efforts will focus on increasing the potency of the systems by exploring other antibiotics, but also their ability to function in biological non-Newtonian fluids, such as mucus or saliva. The sensing capabilities of the MSR-1 will also be further probed, by investigating their oxygen sensitivity to the depleted oxygen zones of the biofilm and determine if MSR-1 biohybrids could detect and target biofilms with minimal magnetic navigation. Finally, increasing the quantity of biohybrids will be investigated to improve the therapeutic capacity of biohybrid drug delivery systems.

\section{MATERIALS AND METHODS}

Fabrication of MSMs. MSMs were synthesized using a cyclopore PC membrane template, containing $2 \mu \mathrm{m}$ maximum diameter conicalshaped micropores (catalog no. 7060-2511; Whatman, Maidstone, UK). Hexadecyltrimethylammonium bromide ( $12.5 \mathrm{mg}$, 99\%, SigmaAldrich) and triethanolamine (10 mg, 98\%, Sigma-Aldrich) were dissolved in $4 \mathrm{~mL}$ of deionized water in a glass vial containing the polycarbonate membrane. The mix was heated to $80^{\circ} \mathrm{C}$, and $5 \mu \mathrm{L}$ of tetraethyl orthosilicate (TEOS, 99\%, Sigma-Aldrich) was added under stirring. After $30 \mathrm{~min}$ at constant temperature, $30 \mu \mathrm{L}$ of a mixture of 1:2 v/v APTES (Sigma-Aldrich) and TEOS was added. The reaction was kept at $80^{\circ} \mathrm{C}$ for $2 \mathrm{~h}$ with stirring. The reaction was removed from heat, and the solution was kept stirring for $20 \mathrm{~min}$ at room temperature. The membrane was removed, rinsed extensively with deionized water, and polished on both membrane sides with a moist cotton swap. The polycarbonate membrane template was dissolved in $1 \mathrm{~mL}$ of $\mathrm{CH}_{2} \mathrm{Cl}_{2}$ for $15 \mathrm{~min}$, and the released MSMs were collected by centrifugation at $14000 \mathrm{rpm}$ for $3 \mathrm{~min}$. MSMs were washed, centrifuged, and collected $2 \times$ in $\mathrm{CH}_{2} \mathrm{Cl}_{2}, 2 \times$ in $\mathrm{EtOH}$, and $2 \times$ in deionized $\mathrm{H}_{2} \mathrm{O}$. The MSMs were stored in deionized water at room temperature.

MSR-1 Culture. The cultivation medium from Heyen and Schüler ${ }^{54}$ was used to grow MSR-1 in Hungate tubes. Ten milliliters of a fully grown culture was centrifuged at $5000 \mathrm{rpm}$ for $10 \mathrm{~min}$, and the resulting pellet was isolated and resuspended at the bottom of a semisolid medium column ( $0.1 \%$ agar). The motile part of the population was isolated due to a band formation process in the column. ${ }^{35}$ Bacteria suspensions with optical densities between 0.07 and 0.1 (NanoPhotometer Pearl at $565 \mathrm{~nm}$ ) were used for the guiding experiments.

Biohybrid Microswimmer Formation. MSR-1 cells were centrifuged $(6000 \mathrm{rpm}, 3 \mathrm{~min})$ and resuspended twice in motility media $\left(0.01 \mathrm{M} \mathrm{K}_{3} \mathrm{PO}_{4}, 67 \mathrm{mM} \mathrm{NaCl}, 0.1 \mathrm{mM}\right.$ ethylenediaminetetraacetic acid, $0.002 \% \mathrm{v} / \mathrm{v}$ Tween-20 (pH adjusted to 7.4)). All experiments with bacteria were performed in motility media unless specified. MSMs were added to MSR-1 in motility media and allowed to incubate at ambient temperature for $15 \mathrm{~min}$ before imaging in glass coverslip bottom Petri dishes (Cellview Cell Culture Dish, Greiner Bio-One). Videos and images were acquired using bright field microscopy with an inverted Leica DMI3000B microscope, Leica DFC3000G camera, and Leica Application Suite v.4.5.0 software. For magnetic maneuverability and group swimming experiments, a custom-made microscope ${ }^{35}$ was utilized to generate magnetic fields at the sample position during microscopy. Briefly, a triaxial Helmholtz coil setup (C-SpinCoil-XYZ, Micro Magnetics Inc.) was used to generate a homogeneous magnetic field inside the region of interest directly above the microscope objective. The sample was illuminated with a red LED, and the images were captured with a CMOS camera at $10 \mathrm{~Hz}(2560 \times 2160$ pixels; NeosCMOS, Andor Technology). For "U-turn" analysis, magnetic fields were generated with four orthogonally oriented iron-core electromagnets. The coils were built onto another custom-made microscope stage to surround a $35 \mathrm{~mm}$ Petri dish and placed onto an inverted Zeiss (Carl Zeiss, Inc., Oberkochen, Germany) Axio Observer A1 microscope with a $20 \times$ $(\mathrm{NA}=0.5)$ objective lens. The input current for the coils was controlled by motor drivers and an Arduino microcontroller board, and the $\sim 6 \mathrm{mT}$ magnetic field strength was calibrated using a Lake Shore Cryotronics (Darmstatdt, Germany) model 460-3-channel gaussmeter. For acquiring swimming velocities, MSR-1 and biohyrids were recorded with a Zeiss Axiocam 503 CCD camera. Tracking of MSR-1 and or biohybrids was performed with ImageJ Fiji 1.49s MTrackJ manual tracking software or an in-house-developed script in Python with the OpenCV library using recorded videos. Thresholding criteria were used to distinguish bacteria and tubes from the background with their positions taken as the center of mass of the contour. Bayesian decision making determined the trajectory of the motors over time.

SEM and TEM Imaging of Bacteria and Biohybrids. Bacteria and tubes were imaged with a Zeiss Ultra 55 Gemini scanning electron microscope using an accelerating voltage of $5 \mathrm{keV}$ and an in-lens detector. To prepare samples for SEM, biohybrids suspended in motility media were allowed to sediment on clean, plasma-etched (1 min argon plasma, Diener Electronic Atto Plasma Cleaner) silicon wafer chips $(5 \times 6 \mathrm{~mm})$ for $1 \mathrm{~h}$ at ambient temperature. For biofilm, $E$. coli were allowed to culture on wafers at $30{ }^{\circ} \mathrm{C}$ for $72 \mathrm{~h}$ and then washed with phosphate-buffered saline (PBS). Wafers were incubated in $2.5 \%$ glutaraldehyde in PBS for $45 \mathrm{~min}$ at $4{ }^{\circ} \mathrm{C}$ and rinsed with PBS, then water. Bacteria were dehydrated in a series of increasing aqueous ethanol concentrations $(30 \%, 50 \%, 70 \%, 90 \%$, and $100 \%)$ for $5 \mathrm{~min}$ in each solution and $10 \mathrm{~min}$ in pure ethanol. Bacteria were further dehydrated and preserved using a series of hexamethyldisilazane (HMDS, Sigma-Aldrich) solutions: 2:1 ethanol/HMDS (15 min), 1:2 ethanol/HMDS (15 min), pure HMDS (15 min). Wafers and bacteria were air-dried for $2 \mathrm{~h}$ followed by sputtering deposition of $3 \mathrm{~nm}$ gold using a Bal-tec MED 020 coating system (Leica). Low-magnification, bright field, TEM images of bacteria and MSMs were acquired with a Zeiss EM 912 Omega at $120 \mathrm{kV}$.

Loading MSMs with CFX (CFX-MSMs). Five $\mathrm{mg} / \mathrm{mL}$ ciprofloxacin (CFX, Sigma-Aldrich) was dissolved in $0.1 \mathrm{M}$ aqueous $\mathrm{HCl}$. Then $60 \mu \mathrm{L}$ of the concentrated CFX solution was added to $540 \mu \mathrm{L}$ of MSMs in water. MSMs with CFX were shaken overnight in a pulse vortex mixer (1500 speed) at ambient temperature. MSMS were collected by centrifugation at $20000 \mathrm{rpm}$ for $6 \mathrm{~min}$ and resuspended twice in motility media. CFX-MSMs were used immediately for CFX release measurements or biohybrid formation.

CFX Release from CFX-MSMs. Freshly made CFX-MSMs suspended in $1 \mathrm{~mL}$ of motility media at $\mathrm{pH} 5.8$ or 7.4 in an Eppendorf tube were centrifuged at $20000 \mathrm{rpm}$ for $6 \mathrm{~min}$. A $400 \mu \mathrm{L}$ amount of supernatant was collected from the tube, without disturbing the CFX-MSMs pellet, and was divided into two $200 \mu \mathrm{L}$ aliquots in a black, flat-bottom, chimney 96-well plate (Greiner Bio-One). Well plates also contained calibration samples of CFX in motility media $(0$, $0.05,0.1,0.3,0.5,0.8$, and $1.0 \mu \mathrm{g} / \mathrm{mL}$ ) at $\mathrm{pH} 5.8$ or 7.4 . Samples' fluorescence intensity was measured with a Tecan Infinite M200 multifunctional reader and Tecan i-control 1.10 software. The excitation wavelength and emission wavelength were set to 280 and $425 \mathrm{~nm}$, respectively. After analysis, the supernatant was returned to the CFX-MSMs Eppendorf tube and gently shaken to break up the microtube pellet. These measurements were repeated for the given time points: $0,0.5,1,3,4,6,7$, and $24 \mathrm{~h}$. Release experiments were repeated in triplicate. The calibration curves (Figure S4) in each measurement were used to calculate the concentration of supernatant in the sample solution at each $\mathrm{pH}$.

E. coli Biofilm Formation. E. coli MG1655 cultured on LB agar plates (Sigma-Aldrich) were transferred to $5 \mathrm{~mL}$ of LB broth (SigmaAldrich) and allowed to divide overnight at $30{ }^{\circ} \mathrm{C}$ and $150 \mathrm{rpm}$. A 50 $\mu \mathrm{L}$ amount of concentrated MG1655 solution was diluted in $5 \mathrm{~mL}$ of fresh LB broth. A diluted E. coli solution was plated in sterile nontissue culture treated 96-well plates (Eppendorf) or Petri dishes with glass bottoms and allowed to culture for 24 or $48 \mathrm{~h}$ at $30{ }^{\circ} \mathrm{C}$ for biofilm formation. LB broth was removed and replaced with motility media. 
For inhibition studies of biofilm formation with CFX, diluted E. coli solutions in LB broth were plated in a 96-well plate with various concentrations of CFX $(0-0.3 \mu \mathrm{g} / \mathrm{mL})$, with $200 \mu \mathrm{L}$ per well. The optical density of each well was measured at $600 \mathrm{~nm}\left(\mathrm{OD}_{600}\right)$ with a BioTek Gen5 Synergy 2 plate reader every $20 \mathrm{~min}$ for $15 \mathrm{~h}$ at $30^{\circ} \mathrm{C}$ with gentle shaking.

Visualization of Biofilms. Confocal imaging was performed with a Nikon Eclipse Ti confocal microscope equipped with an Andor EMCCD camera (iXon Ultra 888, $1024 \times 1024$ sensor format of 13 $\mu \mathrm{m} \times 13 \mu \mathrm{m}$ pixel size, Belfast, UK) and a Yokogawa CSU-W1 spinning disk. A CFI Plan Apo VC 20× $(\mathrm{NA}=0.75)$ and a Plan Apo VC $60 \times$ oil $(\mathrm{NA}=1.4)$ objective lens were used for all $3 \mathrm{D}$ confocal images. Twenty-four hour matured E. coli biofilms cultured on glass bottom Petri dishes were washed once with motility media. For imaging live and dead cells within the biofilm, motility media was removed and the biofilms were incubated with $2 \mu \mathrm{L} / \mathrm{mL}$ of propidium iodide and $2 \mu \mathrm{L} / \mathrm{mL}$ of STYO 9 (Life Technologies) in motility media for $15 \mathrm{~min}$. Dye solution was removed, and the biofilm was stained with $1 \mu \mathrm{M}$ LysoSensor Blue DND-167 (Life Technologies) for $1 \mathrm{~h}$ at $37{ }^{\circ} \mathrm{C}$, followed by gentle washing with fresh motility media. $\mathrm{pH}$ distribution within the biofilm architecture was reconstructed using Nikon NIS AR software (v.4.60). For analysis of biofilms with microtubes, biofilms were incubated with MSMs or CFX-MSMs. Unmodified biofilms were used as a control group. Live/dead labeling and imaging was performed as described previously. A set of biofilms were stained after the initial incubation with MSMs or CFX-MSMs ( $t$ $=0 \mathrm{~h}$ ), and separate biofilm sets were stained $24 \mathrm{~h}$ after incubation with the microtubes for comparison. The staining solution was removed, and the biofilms were rinsed once with motility media. 3D confocal imaging of the biofilms was used to observe each experimental group. To visualize the release of CFX from CFXMSMs, CFX-MSMs were incubated with a $24 \mathrm{~h}$ matured E. coli biofilm. Time-lapsed 3D confocal images of CFX fluorescence were taken over $24 \mathrm{~h}$. Since only CFX fluorescence was imaged, biofilm absorbing the CFX from the CFX-MSMs could be observed.

CV Biofilm Assay. Biofilm CV analysis protocol was adapted from Merrit et al. ${ }^{55}$ Sterile nontissue culture treated 96-well plates (Eppendorf) had $100 \mu \mathrm{L}$ aliquots of bacteria solution placed in each well or LB broth as a control. Biofilm was cultured for $24 \mathrm{~h}$ at $30^{\circ} \mathrm{C}$ in a static environment. LB broth was removed, and each well of the biofilm was washed with $2 \times$ with motility media. The motility media was removed, and wells were incubated with $100 \mu \mathrm{L}$ of biohybrids or experimental control groups in motility media $(0.05 \mu \mathrm{g} / \mathrm{mL}$ CFX, 0.1 $\mu \mathrm{g} / \mathrm{mL}$ CFX, MSR-1, MSMs, MSR-1+MSMs, CFX-MSMs, and CFXMSMs+MSR-1). Unmodified biofilm wells, blank wells, and wells with LB broth were used as controls. Each experimental parameter had five wells. The $\mathrm{OD}_{600}$ of MSR- 1 was kept at $\sim 0.04$ for all experiments. The $\mathrm{OD}_{600}$ was obtained from $200 \mu \mathrm{L}$ of motility media with MSR-1 in a 96-well plate at ambient temperature using bacteria-free media for a control. The density of MSMs and CFX-MSMs for all experimental parameters was kept at $\sim 20000$ microtubes per well. E. coli biofilms were incubated with control groups and biohybrids for $24 \mathrm{~h}$ at $25{ }^{\circ} \mathrm{C}$ in a static environment. Media and controls were removed, and wells were washed once with fresh motility media. A $125 \mu \mathrm{L}$ amount of $0.1 \%$ $\mathrm{w} / \mathrm{v}$ aqueous CV (Sigma-Aldrich) was put in each well and stained the biofilms for $10 \mathrm{~min}$. Stain was removed, and wells were washed $3 \times$ with $150 \mu \mathrm{L}$ of deionized water. Water was removed, and wells were allowed to air-dry for $15 \mathrm{~min}$ followed by incubation with $200 \mu \mathrm{L}$ of $80 \% \mathrm{v} / \mathrm{v}$ ethanol in water for $15 \mathrm{~min}$ to solubilize the biofilm. CV solutions were pipetted into a new 96 -well plate and had their $\mathrm{OD}_{590}$ measured with the BioTek plate reader. The $\mathrm{CV}$ biofilm assay was repeated in triplicate, and statistical significance of the data was evaluated using one-way analysis of variance (ANOVA) followed by post hoc Bonferroni tests to compare individual means using OriginPro 9.10 software. The level of statistically significant data had $P$-values of 0.01 or less when compared to the umodified biofilm group.

\section{ASSOCIATED CONTENT}

\section{Supporting Information}

The Supporting Information is available free of charge on the ACS Publications website at DOI: 10.1021/acsnano.7b04128.

Supporting videos' description and supporting figures (PDF)

Video SV1: Biohybrid: MSR-1 swimming with MSM (AVI)

Video SV2: Biohybrid swimming in a figure 8 guided with external magnetic coils (AVI)

Video SV3: Multiple biohybrids guided by external magnetic coils (AVI)

Video SV4: Time lapse video of 3D confocal images of CFX release from CFX-MSMs (AVI)

Video SV5: Biohybrid guided with permanent magnet to island of E. coli biofilm (AVI)

\section{AUTHOR INFORMATION}

\section{Corresponding Authors}

*E-mail: damien.faivre@mpikg.mpg.de.

*E-mail: sitti@is.mpg.de.

*E-mail: ssanchez@ibecbarcelona.eu.

ORCID

Morgan M. Stanton: 0000-0002-9688-7536

Diana Vilela: 0000-0001-5005-7070

Damien Faivre: 0000-0001-6191-3389

Metin Sitti: 0000-0001-8249-3854

Samuel Sánchez: 0000-0002-5845-8941

\section{Author Contributions}

${ }^{\#}$ M. M. Stanton and B.-W. Park contributed equally to this publication. All authors were involved in conceiving and designing the research plan and experiments. M.M.S. and B.W.P. performed all lab-bench and microscopy imaging experiments of bacteria and biohybrids and were responsible for their data analysis. D.V. was responsible for fabrication of the mesoporous silica microtubes and their quantification. K.B. was responsible for MSR-1 bacteria culture and assisted with imaging bacteria under magnetic fields. M.M.S. wrote the publication with editorial assistance from the other authors. All authors discussed the results and their implications and revised the manuscript and figures at all stages.

\section{Notes}

The authors declare no competing financial interest.

\section{ACKNOWLEDGMENTS}

The research leading to these results was achieved thanks to the financial support from the European Research Council for the European Union's Seventh Framework Program (FP7/20072013) starting grant no. 311529 (Lab-in-a-tube and Biosensors) and the Grassroots Initiative funding from the Max Planck Institute for Intelligent Systems. The authors acknowledge financial support from the Max Planck Society. D.V. acknowledges support from the Alexander von Humboldt Foundation and H2020 COFUND scheme [grant agreement no. 712754] and by the Severo Ochoa programme of MICINN [grant SEV2014-0425]. The authors would also like to thank Mr. Kersten Hahn for assisting with the TEM images of MSMs, Ms. Anna Pohl for assisting with TEM images of the MSR-1, and Mr. Albert Miguel-Lopez for developing the in-house tracking software. 


\section{REFERENCES}

(1) Flemming, H.-C.; Wingender, J. The Biofilm Matrix. Nat. Rev. Microbiol. 2010, 8, 623-633.

(2) Schultz, M. P.; Bendick, J. A.; Holm, E. R.; Hertel, W. M. Economic Impact of Biofouling on a Naval Surface Ship. Biofouling 2011, 27, 87-98.

(3) Rodrigues, L. R. Inhibition of Bacterial Adhesion on Medical Devices. In Bacterial Adhesion; Linke, D.; Goldman, A., Eds.; Springer, 2011; Vol. 715, pp 351-367.

(4) Davies, J.; Davies, D. Origins and Evolution of Antibiotic Resistance. Microbiol. Mol. Biol. Rev. 2010, 74, 417-433.

(5) Levin-Reisman, I.; Ronin, I.; Gefen, O.; Braniss, I.; Shoresh, N.; Balaban, N. Q. Antibiotic Tolerance Facilitates the Evolution of Resistance. Science 2017, 355, 82610.1126/science.aaj2191.

(6) Bryers, J. D. Medical Biofilms. Biotechnol. Bioeng. 2008, 100, 118

(7) Lynch, A. S.; Robertson, G. T. Bacterial and Fungal Biofilm Infections. Annu. Rev. Med. 2008, 59, 415-428.

(8) Høiby, N.; Bjarnsholt, T.; Givskov, M.; Molin, S.; Ciofu, O. Antibiotic Resistance of Bacterial Biofilms. Int. J. Antimicrob. Agents 2010, 35, 322-332.

(9) Lebeaux, D.; Ghigo, J.-M.; Beloin, C. Biofilm-Related Infections: Bridging the Gap between Clinical Management and Fundamental Aspects of Recalcitrance toward Antibiotics. Microbiol. Mol. Biol. Rev. 2014, 78, 510-543.

(10) de Faria, A. F.; Martinez, D. S. T.; Meira, S. M. M.; de Moraes, A. C. M.; Brandelli, A.; Filho, A. G. S.; Alves, O. L. Anti-Adhesion and Antibacterial Activity of Silver Nanoparticles Supported on Graphene Oxide Sheets. Colloids Surf., B 2014, 113, 115-124.

(11) Horev, B.; Klein, M. I.; Hwang, G.; Li, Y.; Kim, D.; Koo, H.; Benoit, D. S. W. pH-Activated Nanoparticles for Controlled Topical Delivery of Farnesol to Disrupt Oral Biofilm Virulence. ACS Nano 2015, 9, 2390-2404.

(12) Tuson, H. H.; Weibel, D. B. Bacteria-Surface Interactions. Soft Matter 2013, 9, 4368-4380.

(13) Forier, K.; Raemdonck, K.; De Smedt, S. C.; Demeester, J.; Coenye, T.; Braeckmans, K. Lipid and Polymer Nanoparticles for Drug Delivery to Bacterial Biofilms. J. Controlled Release 2014, 190, 607-623.

(14) Baelo, A.; Levato, R.; Julián, E.; Crespo, A.; Astola, J.; Gavaldà, J.; Engel, E.; Mateos-Timoneda, M. A.; Torrents, E. Disassembling Bacterial Extracellular Matrix with Dnase-Coated Nanoparticles to Enhance Antibiotic Delivery in Biofilm Infections. J. Controlled Release 2015, 209, 150-158.

(15) Li, J.; Esteban-Fernández de Ávila, B.; Gao, W.; Zhang, L.; Wang, J., Micro/Nanorobots for Biomedicine: Delivery, Surgery, Sensing, and Detoxification. Science Robotics 2017, 2.eaam643110.1126/scirobotics.aam6431

(16) Katuri, J.; Ma, X.; Stanton, M. M.; Sánchez, S. Designing Microand Nanoswimmers for Specific Applications. Acc. Chem. Res. 2017, 50, $2-11$.

(17) Koumakis, N.; Lepore, A.; Maggi, C.; Di Leonardo, R. Targeted Delivery of Colloids by Swimming Bacteria. Nat. Commun. 2013, 4, 2588.

(18) Vizsnyiczai, G.; Frangipane, G.; Maggi, C.; Saglimbeni, F.; Bianchi, S.; Di Leonardo, R. Light Controlled 3D Micromotors Powered by Bacteria. Nat. Commun. 2017, 8, 15974.

(19) Carlsen, R. W.; Sitti, M. Bio-Hybrid Cell-Based Actuators for Microsystems. Small 2014, 10, 3831-3851.

(20) Ghosh, A.; Fischer, P. Controlled Propulsion of Artificial Magnetic Nanostructured Propellers. Nano Lett. 2009, 9, 2243-2245.

(21) Mhanna, R.; Qiu, F.; Zhang, L.; Ding, Y.; Sugihara, K.; ZenobiWong, M.; Nelson, B. J. Artificial Bacterial Flagella for RemoteControlled Targeted Single-Cell Drug Delivery. Small 2014, 10, 1953-1957.

(22) Mair, L. O.; Nacev, A.; Hilaman, R.; Stepanov, P. Y.; Chowdhury, S.; Jafari, S.; Hausfeld, J.; Karlsson, A. J.; Shirtliff, M. E.; Shapiro, B.; Weinberg, I. N. Biofilm Disruption with Rotating
Microrods Enhances Antimicrobial Efficacy. J. Magn. Magn. Mater. 2017, 427, 81-84.

(23) Medina-Sánchez, M.; Schwarz, L.; Meyer, A. K.; Hebenstreit, F.; Schmidt, O. G. Cellular Cargo Delivery: Toward Assisted Fertilization by Sperm-Carrying Micromotors. Nano Lett. 2016, 16, 555-561.

(24) Magdanz, V.; Sanchez, S.; Schmidt, O. G. Development of a Sperm-Flagella Driven Micro-Bio-Robot. Adv. Mater. 2013, 25, 65816588.

(25) Feinberg, A. W.; Feigel, A.; Shevkoplyas, S. S.; Sheehy, S.; Whitesides, G. M.; Parker, K. K. Muscular Thin Films for Building Actuators and Powering Devices. Science 2007, 317, 1366-1370.

(26) Williams, B. J.; Anand, S. V.; Rajagopalan, J.; Saif, M. T. A. A Self-Propelled Biohybrid Swimmer at Low Reynolds Number. Nat. Commun. 2014, 5, 3081.

(27) Raman, R.; Cvetkovic, C.; Uzel, S. G. M.; Platt, R. J.; Sengupta, P.; Kamm, R. D.; Bashir, R. Optogenetic Skeletal Muscle-Powered Adaptive Biological Machines. Proc. Natl. Acad. Sci. U. S. A. 2016, 113, 3497-3502.

(28) Stanton, M. M.; Simmchen, J.; Ma, X.; Miguel-López, A.; Sánchez, S. Biohybrid Janus Motors Driven by Escherichia coli. Adv. Mater. Interfaces 2016, 3, 1500505.

(29) Hosseinidoust, Z.; Mostaghaci, B.; Yasa, O.; Park, B.-W.; Singh, A. V.; Sitti, M. Bioengineered and Biohybrid Bacteria-Based Systems for Drug Delivery. Adv. Drug Delivery Rev. 2016, 106, 27-44.

(30) Martel, S.; Tremblay, C. C.; Ngakeng, S.; Langlois, G. Controlled Manipulation and Actuation of Micro-Objects with Magnetotactic Bacteria. Appl. Phys. Lett. 2006, 89, 233904.

(31) Martel, S.; Mohammadi, M. In A Robotic Micro-Assembly Process Inspired by the Construction of the Ancient Pyramids and Relying on Several Thousand Flagellated Bacteria Acting as Micro-Workers; 2009 IEEE/RSJ International Conference on Intelligent Robots and Systems, 10-15 Oct 2009; 2009; pp 426-427.

(32) Lanauze, D. d.; Felfoul, O.; Turcot, J.-P.; Mohammadi, M.; Martel, S. Three-Dimensional Remote Aggregation and Steering of Magnetotactic Bacteria Microrobots for Drug Delivery Applications. Int. Robot. Res. 2014, 33, 359-374.

(33) Ma, Q.; Chen, C.; Wei, S.; Chen, C.; Wu, L.-F.; Song, T. Construction and Operation of A Microrobot Based on Magnetotactic Bacteria in a Microfluidic Chip. Biomicrofluidics 2012, 6, 024107.

(34) Felfoul, O.; Mohammadi, M.; Taherkhani, S.; de Lanauze, D.; Zhong, X. Y.; Loghin, D.; Essa, S.; Jancik, S.; Houle, D.; Lafleur, M.; Gaboury, L.; Tabrizian, M.; Kaou, N.; Atkin, M.; Vuong, T.; Batist, G.; Beauchemin, N.; Radzioch, D.; Martel, S. Magneto-Aerotactic Bacteria Deliver Drug-Containing Nanoliposomes to Tumour Hypoxic Regions. Nat. Nanotechnol. 2016, 11, 941-947.

(35) Bennet, M.; McCarthy, A.; Fix, D.; Edwards, M. R.; Repp, F.; Vach, P.; Dunlop, J. W. C.; Sitti, M.; Buller, G. S.; Klumpp, S.; Faivre, D. Influence of Magnetic Fields on Magneto-Aerotaxis. PLoS One 2014, 9, e101150.

(36) Lefêvre, C. T.; Bennet, M.; Landau, L.; Vach, P.; Pignol, D.; Bazylinski; Dennis, A.; Frankel; Richard, B.; Klumpp, S.; Faivre, D. Diversity of Magneto-Aerotactic Behaviors and Oxygen Sensing Mechanisms in Cultured Magnetotactic Bacteria. Biophys. J. 2014, $107,527-538$.

(37) Argyo, C.; Weiss, V.; Bräuchle, C.; Bein, T. Multifunctional Mesoporous Silica Nanoparticles as a Universal Platform for Drug Delivery. Chem. Mater. 2014, 26, 435-451.

(38) Stanton, M. M.; Park, B.-W.; Miguel-López, A.; Ma, X.; Sitti, M.; Sänchez, S. Biohybrid Microtube Swimmers Driven by Single Captured Bacteria. Small 2017, 13, 1603679.

(39) Reufer, M.; Besseling, R.; Schwarz-Linek, J.; Martinez, V. A.; Morozov, A. N.; Arlt, J.; Trubitsyn, D.; Ward, F. B.; Poon, W. C. K. Switching of Swimming Modes in Magnetospirillium gryphiswaldense. Biophys. J. 2014, 106, 37-46.

(40) Nadkarni, R.; Barkley, S.; Fradin, C. A Comparison of Methods to Measure the Magnetic Moment of Magnetotactic Bacteria through Analysis of their Trajectories in External Magnetic Fields. PLoS One 2013, 8, e82064. 
(41) Esquivel, D. M. S.; Lins De Barros, H. G. P. Motion of Magnetotactic Microorganisms. J. Exp. Biol. 1986, 121, 153-163.

(42) Fass, R. J. Efficacy and Safety of Oral Ciprofloxacin for Treatment of Serious Urinary Tract Infections. Antimicrob. Agents Chemother. 1987, 31, 148-150.

(43) Hidalgo, G.; Burns, A.; Herz, E.; Hay, A. G.; Houston, P. L.; Wiesner, U.; Lion, L. W. Functional Tomographic Fluorescence Imaging of $\mathrm{pH}$ Microenvironments in Microbial Biofilms by Use of Silica Nanoparticle Sensors. Appl. Environ. Microbiol. 2009, 75, 74267435.

(44) Xiao, J.; Klein, M. I.; Falsetta, M. L.; Lu, B.; Delahunty, C. M.; Yates, J. R., III; Heydorn, A.; Koo, H. The Exopolysaccharide Matrix Modulates the Interaction between 3D Architecture and Virulence of a Mixed-Species Oral Biofilm. PLoS Pathog. 2012, 8, e1002623.

(45) Felfoul, O.; Martel, S. Assessment of Navigation Control Strategy for Magnetotactic Bacteria in Microchannel: Toward Targeting Solid Tumors. Biomed. Microdevices 2013, 15, 1015-1024.

(46) Stanton, M. M.; Sänchez, S. Pushing Bacterial Biohybrids to In Vivo Applications. Trends Biotechnol. 2017, 35, 910.

(47) Houry, A.; Gohar, M.; Deschamps, J.; Tischenko, E.; Aymerich, S.; Gruss, A.; Briandet, R. Bacterial Swimmers that Infiltrate and Take Over the Biofilm Matrix. Proc. Natl. Acad. Sci. U. S. A. 2012, 109, 13088-13093.

(48) Sullivan, Å; Edlund, C.; Nord, C. E. Effect of Antimicrobial Agents on the Ecological Balance of Human Microflora. Lancet Infect. Dis. 2001, 1, 101-114.

(49) Dethlefsen, L.; Huse, S.; Sogin, M. L.; Relman, D. A. The Pervasive Effects of an Antibiotic on the Human Gut Microbiota, as Revealed by Deep 16S rRNA Sequencing. PLoS Biol. 2008, 6, e280.

(50) Sanders, J. W. E. Efficacy, Safety, and Potential Economic Benefits of Oral Ciprofloxacin in the Treatment of Infections. Clin. Infect. Dis. 1988, 10, 528-543.

(51) W, M. C., III; Roe, F.; Bugnicourt, A.; Franklin, M. J.; Stewart, P. S. Contributions of Antibiotic Penetration, Oxygen Limitation, and Low Metabolic Activity to Tolerance of Pseudomonas aeruginosa Biofilms to Ciprofloxacin and Tobramycin. Antimicrob. Agents Chemother. 2003, 47, 317-323.

(52) Kvist, M.; Hancock, V.; Klemm, P. Inactivation of Efflux Pumps Abolishes Bacterial Biofilm Formation. Appl. Environ. Microbiol. 2008, 74, 7376-7382.

(53) Chen, C.; Chen, L.; Wang, P.; Wu, L.-F.; Song, T. MagneticallyInduced Elimination of Staphylococcus Aureus by Magnetotactic Bacteria Under a Swing Magnetic Field. Nanomedicine 2017, 13, 363370.

(54) Heyen, U.; Schüler, D. Growth and Magnetosome Formation by Microaerophilic Magnetospirillum Strains in an Oxygen-Controlled Fermentor. Appl. Microbiol. Biotechnol. 2003, 61, 536-544.

(55) Merritt, J. H.; Kadouri, D. E.; O’Toole, G. A. Growing and Analyzing Static Biofilms. In Current Protocols in Microbiology; John Wiley \& Sons, Inc., 2005. 